\title{
Trade-Offs for Viruses in Overcoming Innate Immunities in Plants
}

\author{
Yuri Miyashita, ${ }^{1}$ Go Atsumi, ${ }^{2}$ and Kenji S. Nakahara ${ }^{1}$ \\ ${ }^{1}$ Pathogen-Plant Interactions Group, Plant Breeding Science, Research Faculty of Agriculture, Hokkaido University, Sapporo \\ 060-8589, Japan; and ${ }^{2}$ National Institute of Advanced Industrial Science and Technology, Sapporo 062-8517, Japan
}

Accepted 7 June 2016.

\begin{abstract}
Plants recognize viral infection via an immune receptor, i.e., nucleotide-binding site (NB)-leucine-rich repeat (LRR) proteins. Another immune receptor, receptor-like kinase proteins, which share an LRR domain with NB-LRRs, perceive conserved molecules of pathogens called pathogen- or microbeassociated molecular patterns, but NB-LRRs generally perceive particular viral proteins. As viruses can evolve more rapidly than the host immune system, how do plant immune systems, which rely on the perception of proteins, remain effective? Viral adaptive evolution may be controlled by penalties that result from mutations in viral proteins that are perceived by NB-LRRs. Our recent studies in pea (Pisum sativum) suggest a penalty of increased susceptibility to another immune system. When a viral protein mutates to evade one immune system, the virus with the mutated protein becomes more susceptible to another. Such antagonistic pleiotropy of a viral protein by two independent plant immune systems may have precedents. Plants may rely on pairs of immune systems to constrain adaptive evolution by viruses and thereby maintain durable antiviral immunity.
\end{abstract}

Organisms are thought to have developed antiviral immune systems through coevolution with viruses. However, these immune systems can never permanently overcome viruses because of the much faster virus evolution due to high error rates of viral replicase, shorter generation times, and larger populations (Duffy et al. 2008). Nonetheless, immune systems remain competitive. It is therefore an interesting question as to how organisms have managed to maintain durable antiviral immune systems, especially plants, which are sessile and, thus, face the same set of pathogens for a long time. Recent studies in pea (Atsumi et al. 2016; Choi et al. 2013) suggest that a virus that overcomes a plant immune system faces a trade-off in reduction of its ability to overcome a parallel immune system in the same plant. Such trade-offs could constrain the adaptive evolution of viruses and partly explain how plant immune systems remain durable. Reconsidering other known antiviral immune systems from this point of view shows that such a trade-off is not unprecedented. This review focuses on paired plant immune systems that could enforce such trade-offs for viruses.

Corresponding author: K. S. Nakahara; E-mail: knakahar@ res.agr.hokudai. ac.jp; Telephone: +81 11706 2490; Fax: +81 117062483 .

() 2016 The American Phytopathological Society
Trade-offs for viruses in overcoming RNA silencing and effector-triggered immunity implied in the zigzag model.

The idea of the trade-off is inspired by the "zigzag" model, which proposes a simplified coevolutionary arms race between innate immunities and pathogenic microbes (Jones and Dangl 2006). This model was based on studies of interactions between plant immunities and bacterial and fungal pathogens. When adapted for plant-virus interactions, the zigzag model implies collaborative actions of layered innate immunities in enforcing trade-offs on viruses.

In the canonical zigzag model, plants have developed layered innate immunities with two kinds of receptors to recognize pathogen infections and mount defense reactions (Boller and Felix 2009). One is a receptor-like kinase (RLK) that is anchored on the plasma membrane of the plant cell and monitors the outside of the membrane. RLKs perceive conserved structures or molecules on microbes called pathogen- or microbeassociated molecular patterns (PAMPs or MAMPs) and induce defense reactions called pattern-triggered immunity (PTI) against diverse pathogenic microbes that invade the apoplast. Thus, RLKs could be functional homologs of animal toll-like receptors (TLR), as both are anchored to the plasma membrane and share a leucine-rich repeat (LRR) domain that is mainly involved in the perception of MAMPs (Padmanabhan et al. 2009). However, no RLKs that recognize viral infection have been identified so far, though an immune receptor that is supposed to be an RLK dependent on the somatic embryogenesis RLK1 (SERK1) perceives double-stranded RNA (dsRNA) as viral MAMPs to induce PTI, separate from RNA silencing (Niehl et al. 2016). Instead, there is ample evidence for the recognition of viral infection by another class of plant immune receptor, a nucleotide binding site (NB)-LRR protein (Moffett 2009). Although possessing an LRR domain, NB-LRRs do not perceive MAMPs but do, generally, perceive a particular protein of pathogens in the cytoplasm and induce strong defense reactions and programmed cell death, called the hypersensitive response (HR). This specificity - that is, HR is induced only when the plant produces an NB-LRR that perceives the protein produced by pathogen-is described by the gene-for-gene theory (Flor 1971). If the NB-LRR-perceived protein was dispensable for a pathogen, the pathogen could easily evade eliciting the NBLRR-mediated HR by discarding the protein. However, the HR defense systems involving specific perception by NB-LRR are durable enough to be used for breeding crops to confer resistance to diverse pathogens. The zigzag model gives a feasible reason for their durability, i.e., the NB-LRR perceives a pathogen protein that suppresses or circumvents PTI. This protein is called the effector and the NB-LRR-mediated HR 
defense is called effector-triggered immunity (ETI). In the model, by discarding the effector and evading ETI in a host, the pathogen sacrifices its ability to suppress PTI, resulting in effective control of the pathogen by PTI. These effects of antagonistic pleiotropy of the pathogen effector on PTI (Fig. 1A, effector-suppressed immunity [ESI]) and ETI cause the trade-off in overcoming both immune systems (Fig. 1A and B). As such, trade-offs are not necessarily inevitable for bacterial and fungal pathogens, which usually have several effectors and can discard the effector that is perceived by the NB-LRR without losing their ability to suppress PTI, the zigzag model might not have definitively explained the trade-offs.

In contrast, plant viral proteins that are perceived by NB-LRRs are multifunctional and essential. Most plant viruses are RNA viruses, and these, typically, have small genome sizes (generally around $10 \mathrm{~kb}$ but up to 20 ) that encode from three up to only a dozen or so proteins that must perform several functions. Therefore, if a viral protein that is perceived by NB-LRR suppresses PTI, the protein is thus an effector for which antagonistic pleiotropy could form the basis for trade-offs that would achieve evolutionary control of the virus. RNA silencing that is induced by dsRNA is considered to work for plant PTI against viruses (Nakahara and Masuta 2014). Consistent with this, most plant viruses produce proteins that suppress RNA silencing (RNA silencing suppressor [RSS]) in infected plant cells (Csorba et al. 2015). In the zigzag model, an RSS could be, then, considered to be a viral effector. In fact, several plants perceive viral RSSs and induce HR or extreme resistance, which is thought to be an enhanced version of HR that completely prevents viral multiplication and is not associated with programmed cell death (Nakahara and Masuta 2014). Moreover, recent studies provide evidence that NB-LRRs perceive the suppression activity of
RSSs (de Ronde et al. 2014; Wang et al. 2015). The greater the RSS activity, the more strongly HR against that RSS appears to be induced. RNA silencing via micro-RNAs controls the expression of many NB-LRRs (Shivaprasad et al. 2012; Zhai et al. 2011), which appears to be one of the reasons for the correlation between RSS activity and the strength of HR. These studies indicate antagonistic pleiotropy of at least the action of some viral RSSs in RNA silencing and ETI.

\section{Other pairs of independent antiviral immune systems causing trade-offs.}

In addition to RNA silencing and ETI targeting of viral RSSs, other combinations may impose antagonistic pleiotropy on viral effectors in plants. For example, the Cynl-and cyvl-mediated independent immune systems against Clover yellow vein virus (ClYVV) in peas may be another combination (Atsumi et al. 2016; Choi et al. 2013). What these genes encode remains to be identified, but both products interact with CIYVV P3N-PIPO, which is involved in cell-to-cell movement. Cynl governs systemic lethal necrosis of pea plants in response to CIYVV infection. Evidence from genetic analyses of pea and ClYVV and pharmacological studies (Atsumi et al. 2009, 2016; Ravelo et al. 2007) implies that Cynl encodes an NB-LRR that perceives CIYVV P3N-PIPO and induces systemic HR, resulting in lethal necrosis. This would make the Cynl-mediated immunity an instance of ETI, in which the effector is P3N-PIPO. On the other hand, the efficiency of cyvl-mediated resistance to CIYVV strains was recently shown to vary from immunity to delay of systemic infection (Atsumi et al. 2016). Considering that P3NPIPO is both quantitatively and qualitatively involved in CIYVV virulence in pea carrying cyvl (Choi et al. 2013), Atsumi et al. (2016) interpret the relationship of the Cynl-and cyvl-mediated
A

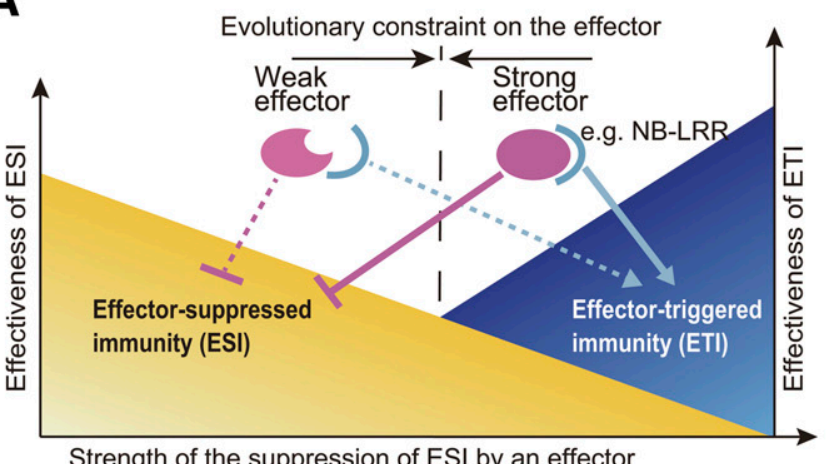

B
C

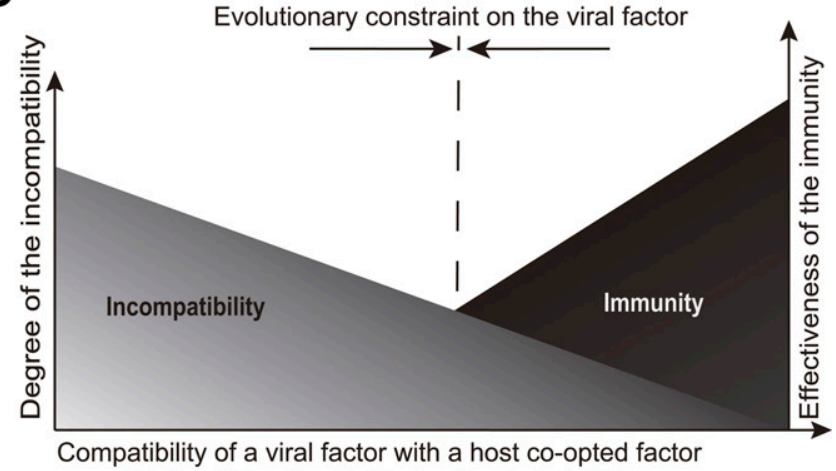

or activity/ stability of a viral factor for viral viability

\begin{tabular}{llll}
\hline ESI & ETI & Viral effector & Reference \\
\hline PTI & NB-LRRs & Effector to suppress PTI & Jones and Dangl, 2006 \\
RNA silencing & NB-LRRs & RNA silencing suppressor & Nakahara and Masuta, 2014 \\
RNA silencing & rgs-CaM & RNA silencing suppressor & Nakahara et al., 2012 \\
RNA silencing & $t m-1$ & Replicase of tobamoviruses & Ishibashi et al., 2011 \\
cyv1 & Cyn1 & P3N-PIPO of ClYVV & Choi et al., 2013; Atsumi et al., 2016
\end{tabular}

Fig. 1. Model of constraint on plant virus evolution. A, Two independent immune systems impose an antagonistic pleiotropy on a viral effector, imposing a trade-off on a virus in overcoming them. When the viral effector mutates to evade effector-triggered immunity (ETI), its ability to overcome effectorsuppressed immunity (ESI) is reduced. B, Recent studies (Atsumi et al. 2016; Choi et al. 2013; Jones and Dangl 2006; Ishibashi et al. 2011; Nakahara and Masuta 2014; Nakahara et al. 2012) show various ETI-ESI combinations that impose antagonistic pleiotropy on viral effectors. These results suggest that this is a general strategy of plants to counteract viruses, which evolve much more rapidly than the host plants. C, Immune systems appear to recognize critical domains or activities of viral proteins not only for suppression of ESI but, also, for viral compatibility with the host plant. When a viral protein mutates to evade the immunity, its compatibility with the host plant is reduced. Thus, immunity and incompatibility impose antagonistic pleiotropy on the viral protein (GarcíaArenal and Fraile 2013). This antagonistic pleiotropy of viral effectors or proteins would constrain adaptive virus evolution, maintaining the durability of immune systems against viruses. 
immune systems with CIYVV via its P3N-PIPO to mean that the more effectively P3N-PIPO works in breaking cyvl-mediated resistance, the stronger $C y n 1$ perceives it and induces necrosis. Therefore, these two independent immune systems in pea appear to impose an antagonistic pleiotropy on P3N-PIPO. cyvl is not likely to be associated with antiviral RNA silencing, because the resistance of cyvl is highly specific to ClYVV and related potyviruses and is recessive; pea plants homozygous for $c y v 1$ resist ClYVV but heterozygotes do not. Moreover, transient expression assay with agroinfiltration did not detect RSS activity of CIYVV P3N-PIPO in transgenic Nicotiana benthamiana 16c (G. Atsumi unpublished). Therefore, these studies suggest that ETI and another independent immune system, apart from RNA silencing, are also able to impose trade-offs via antagonistic pleiotropy of a viral effector (Fig. 1B).

In addition, antiviral RNA silencing and two other reported immune systems targeting viral RSSs that seem not to involve NB-LRR could impose trade-offs on viruses. One system is tm1 -mediated resistance in tomato. Tm- 1 encodes a protein involved in resistance to Tomato mosaic virus (ToMV) (Ishibashi et al. 2007); the protein specifically binds to and inhibits ToMV replicase. The $t m-1$-encoded protein does not inhibit the ToMV replicase but does inhibit replicases of other nonadapted tobamoviruses (Ishibashi et al. 2009). Tobamovirus replicases are viral RSSs (Ishibashi et al. 2011). The RSS activity of the replicase of a tobamovirus, Tobacco mild green mosaic virus, was reduced when the virus overcame $\mathrm{tm}$ - 1 -mediated resistance via mutations in the replicase (Ishibashi et al. 2011). This finding implies that the $t m$-1-encoded protein has affinity with a domain or structure of the replicase that is critical for the RSS activity.

Another immune system is mediated by a calmodulin-like protein, rgs-CaM. rgs-CaM directly interacts with various viral RSSs (Anandalakshmi et al. 2000; Nakahara et al. 2012) and is suggested to function in antiviral defense by directing degradation of its binding RSSs by autophagy (Nakahara et al. 2012). Many viral RSSs have been shown to bind to small or long dsRNAs, and this binding seems to be critical for RSS activity (Csorba et al. 2015). The binding domain of rgs-CaM appears to be negatively charged and to have affinity for a positively charged dsRNA binding site of RSSs. The affinity of rgs-CaM for the $2 \mathrm{~b}$ protein, RSS of $\mathrm{Cu}$ cumber mosaic virus, was reduced by a point mutation that disrupted the ability of $2 b$ to bind to dsRNA and, thus, to suppress RNA silencing (Goto et al. 2007). The affinity of rgs-CaM and the tm-1-encoded protein for RSSs can cause antagonistic pleiotropy of viral RSSs. The existence of various combinations of innate immune systems imposing trade-offs on viruses implies that this is a general antiviral defense strategy of plants that maintains the durability of their immune systems (Fig. 1A and B).

\section{Reduced compatibility to host plants is another trade-off for viruses in overcoming one immunity.}

Various viral proteins, likely not RSSs, elicit HR, probably via their perception by NB-LRRs (Moffett 2009). How is the durability of this NB-LRR-mediated ETI secured? Compared with parental viruses that were sensitive to ETI, resistancebreaking viruses showed attenuated virulence, lower multiplication, and virion instability in susceptible hosts (Elena et al. 2014; García-Arenal and Fraile 2013). One feasible reason is that these NB-LRRs perceive a domain or structure of a viral protein that is critical for its enzymatic activity, virion stability, or interaction with co-opted host factors that are required for viral multiplication, cell-to-cell transfer, or longdistance movement. When a virus adaptively evolves to evade perception by NB-LRRs, it bears fitness penalties, resulting in its attenuation in susceptible plants. These penalties could be the trade-offs for overcoming plant immunity (Fig. 1C)
(García-Arenal and Fraile 2013) and could contribute to the durability of the immunity.

Molecular mechanisms underlying these penalties are largely elusive. As Cyn1 perceives P3N-PIPO, which is not an RSS but functions as an effector for $c y v 1$-mediated resistance, part of the penalty or incompatibility might be due to unknown immune systems. It is possible that some viral proteins, which are perceived by NB-LRRs, suppress the PTI via perception of viral dsRNAs by an RLK with SERK1 (Niehl et al. 2016) and that this canonical PTI-ETI imposes trade-offs on the virus. Actually, an RLK, NIK1-mediated antiviral immunity, which involves translation repression, is suppressed by NSP1 of begomoviruses (Zorzatto et al. 2015) and NSP1 is presumed to be perceived by NB-LRRs to induce HR (Garrido-Ramirez et al. 2000; Hussain et al. 2005). Moreover, this HR is suppressed by TrAP, an RSS of Tomato leaf curl New Delhi virus (Hussain et al. 2007). A coat protein of Turnip crinkle virus, which is perceived by an Arabidopsis NB-LRR, HRT, suppresses not only RNA silencing but, also, the basal resistance via binding to a NAC transcription factor, TIP (Donze et al. 2014). We here additionally note host factors, which interact with viral RSSs and are implicated in antiviral immunities, apart from RNA silencing, i.e., RNA binding proteins ALY (Canto et al. 2006), Arabidopsis catalase3 (Inaba et al. 2011), and components of 20S proteasome (Ballut et al. 2005; Sahana et al. 2012). They interact with P19 of Tomato bushy stunt virus, Cucumber mosaic virus $2 \mathrm{~b}$, and potyviral HC-Pro, respectively, and perhaps impose the trade-offs with RNA silencing.

\section{Perspectives.}

There might be hidden NB-LRRs that no longer perceive effectors, since current viruses have already been adaptively evolved to evade them. However, if these NB-LRRs impose tradeoffs on viruses with counterparts such as RNA silencing and cyvlmediated resistance, they would be still effective for preventing the emergence of virulent viruses that strongly suppress the counterparts (Fig. 1A), in other words, keeping viruses attenuated.

In summary, the imposition of trade-offs by plant immune systems that we have described here would seem to be a general strategy by which host plants maintain the durability of their immune systems and evolutionary control of viruses, which evolve much more rapidly than the host plants. When we find an interaction of a virus with an immune system, we should keep in mind the possible involvement of another in imposing trade-offs on the virus.

\section{ACKNOWLEDGMENTS}

We thank P. Palukaitis and K. Kobayashi for critical reading of this manuscript. This work was supported by the Japan Society for the Promotion of Science KAKENHI (no. 25450055 and $16 \mathrm{H} 04879$ to K. S. Nakahara and 25850030 to G. Atsumi), the NOVARTIS Foundation Japan for the Promotion of Science (to K. S. Nakahara), and the Asahi Glass Foundation (to K. S. Nakahara).

\section{LITERATURE CITED}

Anandalakshmi, R., Marathe, R., Ge, X., Herr, J. M., Jr., Mau, C., Mallory, A., Pruss, G., Bowman, L., and Vance, V. B. 2000. A calmodulin-related protein that suppresses posttranscriptional gene silencing in plants. Science 290:142-144.

Atsumi, G., Kagaya, U., Kitazawa, H., Nakahara, K. S., and Uyeda, I. 2009. Activation of the salicylic acid signaling pathway enhances Clover yellow vein virus virulence in susceptible pea cultivars. Mol. PlantMicrobe Interact. 22:166-175.

Atsumi, G., Suzuki, H., Miyashita, Y., Cho, S. H., Hisa, Y., Rihei, S., Shimada, R., Jeon, E. J., Abe, J., Nakahara, K. S., and Uyeda, I. 2016. $\mathrm{P} 3 \mathrm{~N}-\mathrm{PIPO}$, a frameshift product from $P 3$, pleiotropically determines the virulence of clover yellow vein virus in both resistant and susceptible peas. J. Virol. doi: 10.1128/JVI.00190-16. Published online. 
Ballut, L., Drucker, M., Pugnière, M., Cambon, F., Blanc, S., Roquet, F., Candresse, T., Schmid, H. P., Nicolas, P., Gall, O. L., and Badaoui, S. 2005. HcPro, a multifunctional protein encoded by a plant RNA virus, targets the $20 \mathrm{~S}$ proteasome and affects its enzymic activities. J. Gen. Virol. 86:2595-2603.

Boller, T., and Felix, G. 2009. A renaissance of elicitors: Perception of microbe-associated molecular patterns and danger signals by patternrecognition receptors. Annu. Rev. Plant Biol. 60:379-406.

Canto, T., Uhrig, J. F., Swanson, M., Wright, K. M., and MacFarlane, S. A. 2006. Translocation of Tomato bushy stunt virus P19 protein into the nucleus by ALY proteins compromises its silencing suppressor activity. J. Virol. 80:9064-9072.

Choi, S. H., Hagiwara-Komoda, Y., Nakahara, K. S., Atsumi, G., Shimada, R., Hisa, Y., Naito, S., and Uyeda, I. 2013. Quantitative and qualitative involvement of P3N-PIPO in overcoming recessive resistance against Clover yellow vein virus in pea carrying the cyv1 gene. J. Virol. 87: 7326-7337.

Csorba, T., Kontra, L., and Burgyan, J. 2015. Viral silencing suppressors: Tools forged to fine-tune host-pathogen coexistence. Virology 479-480: 85-103.

de Ronde, D., Pasquier, A., Ying, S., Butterbach, P., Lohuis, D., and Kormelink, R. 2014. Analysis of Tomato spotted wilt virus NSs protein indicates the importance of the N-terminal domain for avirulence and RNA silencing suppression. Mol. Plant Pathol. 15:185-195.

Donze, T., Qu, F., Twigg, P., and Morris, T. J. 2014. Turnip crinkle virus coat protein inhibits the basal immune response to virus invasion in Arabidopsis by binding to the NAC transcription factor TIP. Virology 449:207-214.

Duffy, S., Shackelton, L. A., and Holmes, E. C. 2008. Rates of evolutionary change in viruses: Patterns and determinants. Nat. Rev. Genet. 9:267-276.

Elena, S. F., Fraile, A., and García-Arenal, F. 2014. Evolution and emergence of plant viruses. Adv. Virus Res. 88:161-191.

Flor, H. H. 1971. Current status of gene-for-gene concept. Annu. Rev. Phytopathol. 9:275-296.

García-Arenal, F., and Fraile, A. 2013. Trade-offs in host range evolution of plant viruses. Plant Pathol. 62:2-9.

Garrido-Ramirez, E. R., Sudarshana, M. R., Lucas, W. J., and Gilbertson, R. L. 2000. Bean dwarf mosaic virus BV1 protein is a determinant of the hypersensitive response and avirulence in Phaseolus vulgaris. Mol. Plant-Microbe Interact. 13:1184-1194.

Goto, K., Kobori, T., Kosaka, Y., Natsuaki, T., and Masuta, C. 2007. Characterization of silencing suppressor $2 \mathrm{~b}$ of cucumber mosaic virus based on examination of its small RNA-binding abilities. Plant Cell Physiol. 48:1050-1060.

Hussain, M., Mansoor, S., Iram, S., Fatima, A. N., and Zafar, Y. 2005. The nuclear shuttle protein of Tomato leaf curl New Delhi virus is a pathogenicity determinant. J. Virol. 79:4434-4439.

Hussain, M., Mansoor, S., Iram, S., Zafar, Y., and Briddon, R. W. 2007. The hypersensitive response to tomato leaf curl New Delhi virus nuclear shuttle protein is inhibited by transcriptional activator protein. Mol. Plant-Microbe Interact. 20:1581-1588.

Inaba, J., Kim, B. M., Shimura, H., and Masuta, C. 2011. Virus-induced necrosis is a consequence of direct protein-protein interaction between a viral RNA-silencing suppressor and a host catalase. Plant Physiol. 156: 2026-2036.
Ishibashi, K., Masuda, K., Naito, S., Meshi, T., and Ishikawa, M. 2007. An inhibitor of viral RNA replication is encoded by a plant resistance gene. Proc. Natl. Acad. Sci. U.S.A. 104:13833-13838.

Ishibashi, K., Meshi, T., and Ishikawa, M. 2011. Gaining replicability in a nonhost compromises the silencing suppression activity of Tobacco mild green mosaic virus in a host. J. Virol. 85:1893-1895.

Ishibashi, K., Naito, S., Meshi, T., and Ishikawa, M. 2009. An inhibitory interaction between viral and cellular proteins underlies the resistance of tomato to nonadapted tobamoviruses. Proc. Natl. Acad. Sci. U.S.A. 106: 8778-8783.

Jones, J. D., and Dangl, J. L. 2006. The plant immune system. Nature 444: 323-329.

Moffett, P. 2009. Mechanisms of recognition in dominant $R$ gene mediated resistance. Adv. Virus Res. 75:1-33.

Nakahara, K. S., and Masuta, C. 2014. Interaction between viral RNA silencing suppressors and host factors in plant immunity. Curr. Opin. Plant Biol. 20:88-95.

Nakahara, K. S., Masuta, C., Yamada, S., Shimura, H., Kashihara, Y., Wada, T. S., Meguro, A., Goto, K., Tadamura, K., Sueda, K., Sekiguchi, T., Shao, J., Itchoda, N., Matsumura, T., Igarashi, M., Ito, K., Carthew, R. W., and Uyeda, I. 2012. Tobacco calmodulin-like protein provides secondary defense by binding to and directing degradation of virus RNA silencing suppressors. Proc. Natl. Acad. Sci. U.S.A. 109:10113-10118.

Niehl, A., Wyrsch, I., Boller, T., and Heinlein, M. 2016. Double-stranded RNAs induce a pattern-triggered immune signaling pathway in plants. New Phytol. Published online. doi:10.1111/nph.13944

Padmanabhan, M., Cournoyer, P., and Dinesh-Kumar, S. P. 2009. The leucine-rich repeat domain in plant innate immunity: A wealth of possibilities. Cell. Microbiol. 11:191-198.

Ravelo, G., Kagaya, U., Inukai, T., Sato, M., and Uyeda, I. 2007. Genetic analysis of lethal tip necrosis induced by Clover yellow vein virus infection in pea. J. Gen. Plant Pathol. 73:59-65.

Sahana, N., Kaur, H., Basavaraj, Tena, F., Jain, R. K., Palukaitis, P., Canto, T., and Praveen, S. 2012. Inhibition of the host proteasome facilitates papaya ringspot virus accumulation and proteosomal catalytic activity is modulated by viral factor HcPro. PLoS One 7:e52546.

Shivaprasad, P. V., Chen, H. M., Patel, K., Bond, D. M., Santos, B. A., and Baulcombe, D. C. 2012. A microRNA superfamily regulates nucleotide binding site-leucine-rich repeats and other mRNAs. Plant Cell 24:859874.

Wang, K. D., Empleo, R., Nguyen, T. T., Moffett, P., and Sacco, M. A. 2015. Elicitation of hypersensitive responses in Nicotiana glutinosa by the suppressor of RNA silencing protein P0 from poleroviruses. Mol. Plant Pathol. 16:435-448.

Zhai, J., Jeong, D. H., De Paoli, E., Park, S., Rosen, B. D., Li, Y., González, A. J., Yan, Z., Kitto, S. L., Grusak, M. A., Jackson, S. A., Stacey, G., Cook, D. R., Green, P. J., Sherrier, D. J., and Meyers, B. C. 2011. MicroRNAs as master regulators of the plant NB-LRR defense gene family via the production of phased, trans-acting siRNAs. Genes Dev. 25:2540-2553.

Zorzatto, C., Machado, J. P., Lopes, K. V., Nascimento, K. J., Pereira, W. A., Brustolini, O. J., Reis, P. A., Calil, I. P., Deguchi, M., Sachetto-Martins, G., Gouveia, B. C., Loriato, V. A., Silva, M. A., Silva, F. F., Santos, A. A., Chory, J., and Fontes, E. P. 2015. NIK1-mediated translation suppression functions as a plant antiviral immunity mechanism. Nature 520:679-682. 